\title{
LUMBOSACRAL TRANSITIONAL VERTEBRA ASSOCIATED WITH SACRAL SPINA BIFIDA OCCULTA: A CASE REPORT
}

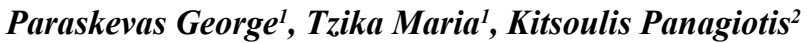

Medical School, Aristotle University of Thessaloniki, Thessaloniki, Greece: Department of Anatomy' ${ }^{1}$, Medical School, University of Ioannina, Greece: Department of Anatomy-Histology-Embryology ${ }^{2}$

Summary: Congenital malformations such as lumbosacral transitional vertebrae and spina bifida occulta constitute unrare anomalies and could affect the symptomatology of low back pain. A transitional vertebra is characterized by elongation of one or both transverse processes, leading to the appearance of a sacralized fifth lumbar vertebra or a lumbarized first sacral vertebra. Furthermore, sacral spina bifida occulta is a developmental anomaly that corresponds to the incomplete closure of the vertebral column. In the present case report, we describe a case of a dried sacrum presenting a partially sacralized fifth lumbar vertebra and total spina bifida, extended from first to fifth sacral vertebra. A pseudoarthrosis is formed on the left side and the specimen could be incorporated in Castellvi's type IIa. Moreover, the incidence, morphology, clinical and surgical significance of these spinal malformations are discussed.
\end{abstract}

Key words: Lumbosacral transitional vertebra; Spina bifida; Bertolotti's syndrome

\section{Introduction}

Developmental anomalies of the spine are common and the clinical impact of their presence is a matter of debate in the literature. Lumbosacral transitional vertebrae (LSTV) consistute congenital vertebral anomalies of the fifth lumbar (L5) - first sacral (S1) vertebra junction. The morphology of a transitional vertebra is a combination of both upper and lower vertebral segments features, leading to a lumbarized S1 or a sacralized L5 vertebra. S1 lumbarization is characterized by nonfusion of S1 and S2 vertebrae, thus the lumbar spine consists of six vertebrae, while a sacralized L5 vertebra is partially or totally fused with the sacrum. The morphology of the transverse process of an LSTV is transformed, causing alterations in the biomechanics of the spine and potentially leading to nerve-root symptomatology (1). Additionally, sacral spina bifida occulta (SSBO) is a term that corresponds to the incomplete closure of sacral canal. The condition may refer to a range of anomalies, varying from partial defect of the posterior arch of some vertebrae to pan-sacral S1-S5 spina bifida (2).

In this case report, we describe a case of a dried sacrum specimen that presented partially sacralized L5 vertebra coexistent with total SSBO. The morphologic variability, clinical and surgical impact of the variant are discussed.

\section{Methods and results}

In a macerated sacrum partially fused with the L 5 vertebra of a male cadaver of Caucasian origin and unknown age, derived from the osteological collection of the Department of Anatomy, a distinct morphologic variability was presented. In particular, the sacrum demonstrated total SSBO, as the posterior S1-S5 laminae showed extended defect. The distances between the unfused sides of the unformed arch ranged from 3mm (S2) to $23 \mathrm{~mm}$ (S1) (Fig. 1). Additionally, L5 was found partially fused with the basis of the sacrum and presented an enlarged dysplastic left transverse process. A pseudoarthrosis was observed on the medial portion of the left side of the enlarged transverse process, whereas on the lateral portion a partial synostosis was detected. Furthermore, a sacrococcygeal synostosis was encountered (Fig. 2). After careful observation of the studied bone, neither pathological conditions such as tumors, nor traumas such as fractures was documented. The combination of the aforementioned variants was documented by photographs, whilst the measurements were made with a sliding digital vernier caliper with an accuracy of $0.1 \mathrm{~mm}$.

\section{Discussion}

LSTV was found present in 7.4-35.6\% (2-6) of the general population in various studies in the literature. Kamanli et al. found that in $37.8 \%$ of the LSTV cases and $2.8 \%$ of the healthy population, the dysplastic transverse process was unilateral, whereas in 8 subjects, thus in approximately $0.02 \%$ of the population, LSTV and SSBO coexisted (3). The existence of SSBO alone was observed in 12.4-21.4\% $(2,3)$ in healthy populations. Eubanks et al found that in only $9.8 \%$ of the SSBO cases, the defect extended from S1 to $\mathrm{S} 5$, thus total SSBO was present in approximately $1.2 \%$ of the population studied (2). 


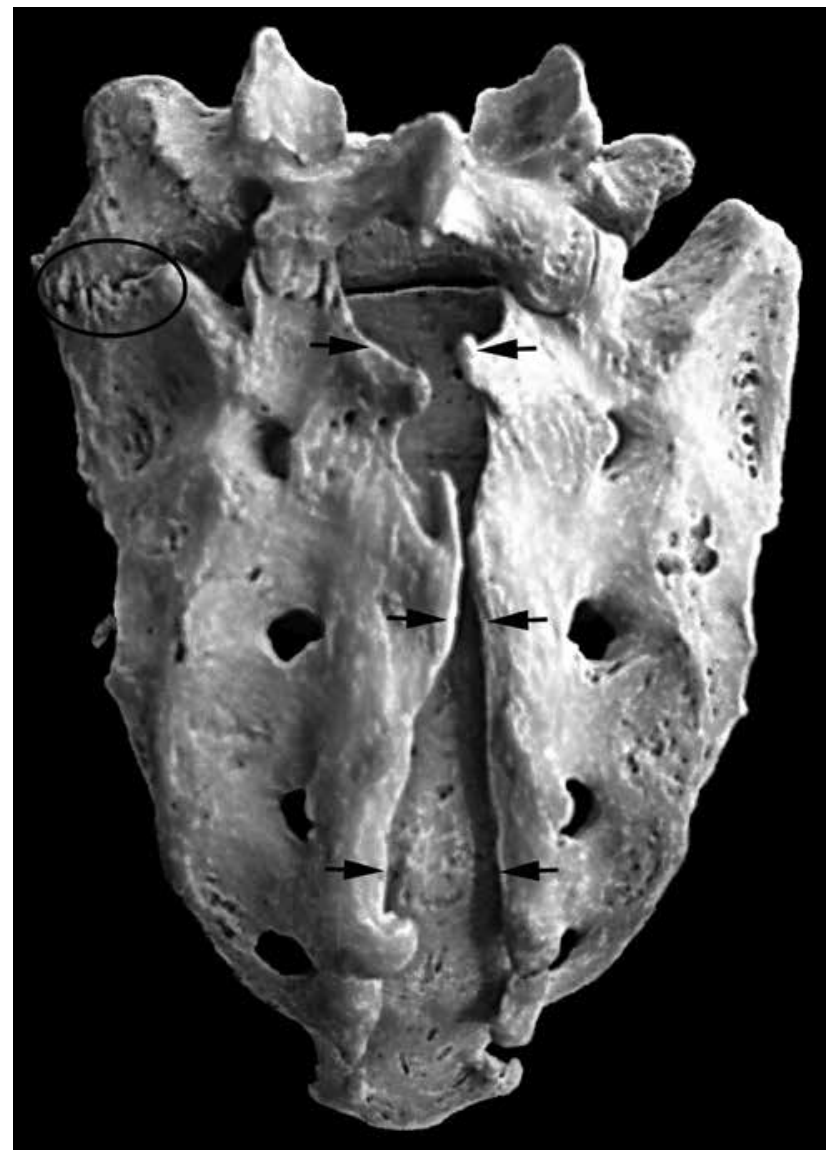

Fig. 1: Posterior aspect of the partially fused sacrum and fifth lumbar vertebra (L5). The arrows demonstrate the incomplete fusion of the posterior vertebral laminae of the sacrum (spina bifida occulta). The partially sacralized L5 vertebra and its enlarged transverse process fused with the sacral basis with pseudoarthrosis and synostosis is also shown on the left side (circle).

Castellvi et al. in 1982 classified the variant LSTVs in four types: in type I, a dysplastic large and triangular in shape transverse process is observed unilaterally (Type Ia) or bilaterally (Type Ib), in type II incomplete lumbarization or sacralization is present unilaterally (Type IIa) or bilaterally (Type IIb), in type III complete lumbarization or sacralization exists unilaterally (Type IIIa) or bilaterally (Type IIIB), while in type IV a mixed complete and incomplete on the other side lumbarization or sacralization is present (1). Our case could be incorporated in Type IIa, as a pseudoarthrosis was observed on the left side and the observed synostosis was partial. Type IIa was found in $12 \%(6)-20 \%$ (1) of the cases diagnosed with LSTV.

The correlation between congenital anomalies of the sacrum and low back pain is obscure. In 1917, Bertolotti stated that the existence of LSTV may be responsible for

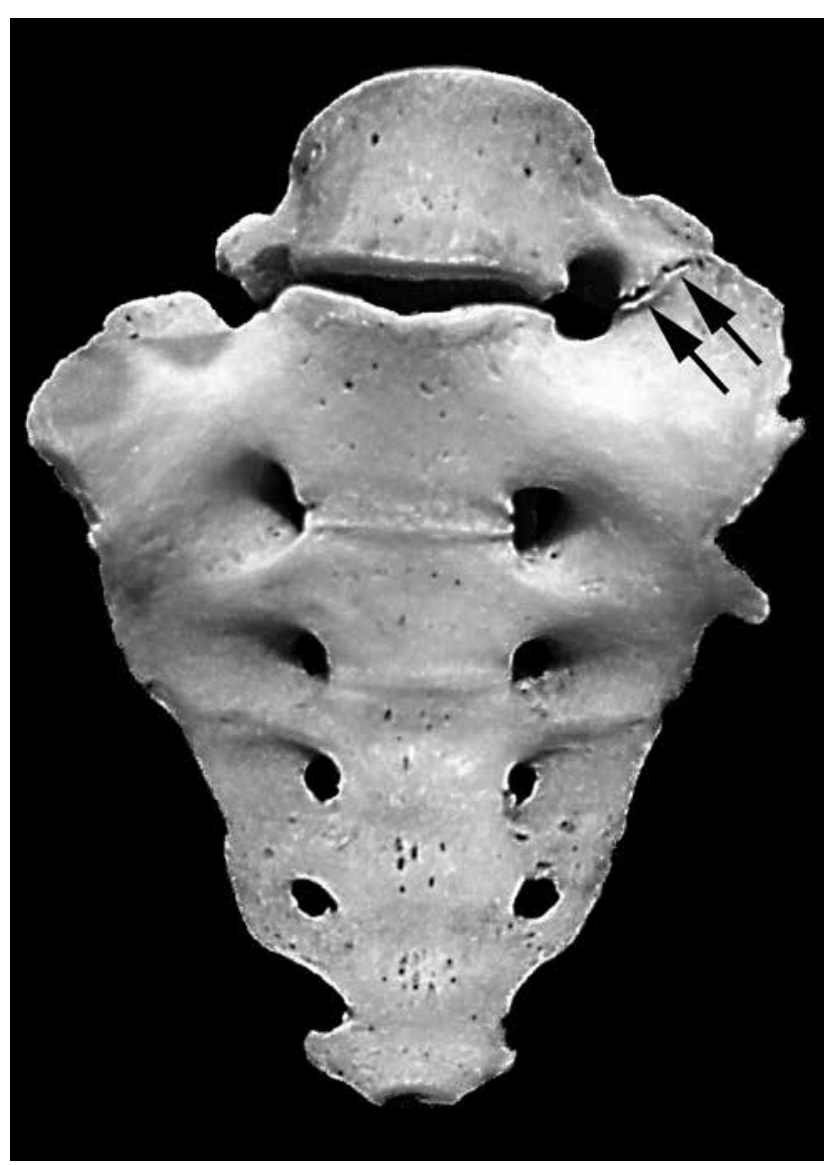

Fig. 2: Anterior aspect of the sacrum and partially fused fifth lumbar vertebra (L5). The formation of the pseudoarthrosis and synostosis between the elongated left transverse process of the L5 vertebra is demonstrated (arrows). This aspect of the sacrum has no anomalies.

unexplained low back pain due to modifications of the biomechanics of the lumbar spine (7). Chronic, persistent low back pain and radiographically identified LSTV constitute the characteristics of Bertolotti's syndrome. During physical examination, tenderness may appear over the region of the lumbar spine and sacrum, whereas conservative treatment includes injections with local anaesthetic and steroid, anti-inflammatory drugs, physiotherapy and exercises (8). In cases with unresolved symptomatology, surgical treatment could provide a solution $(9,10)$.

In $4.5-9.1 \%$ (11-14) of patients experiencing low back pain, the presence of LSTV was detected radiographically. In the study of Taskaynatan et al. another $4.3 \%$ of the patients with low back pain was diagnosed with SSBO, while 2 cases of coexistent LSTV and SSBO were also noticed, corresponding to $0.2 \%$ of the population studied (12). Con- 
sequently, the association of LSTV and SSBO observed in our case is found in $0.02 \%$ of the healthy population (3) and in $0.2 \%$ of population with low back pain (12). The latter authors concluded that these developmental malformations certainly aggravate the clinical severity of the condition, even though their existence may be incidental (12). The presence of LSTV however is characterized as a potential causative factor in patients younger than 30 years old (14). Causal relationship between low back pain and LSTV or SSBO has also been suggested in children population, emerging from the fact that the consequent modifications on the vertebral dynamic segment L5-S1 can lead to degeneration of the intervertebral disc (15).

Otani et al. proposed that the presence of LSTV could influence the appearance of nerve-root symptoms of patients with disc herniation or spinal stenosis without spondylolisthesis, as in most cases, the symptomatic disc was found to be above the LSTV junction; however, the authors clarified that the LSTV presence affects the incidence of symptomatology only in coexistence with spinal pathologies (4). The incidence of structural pathology was not found higher in cases of LSTV by Elster, too, although disc herniation, spinal and nerve root canal stenosis were encountered more often at the level above the LSTV (13). Particularly in LSTV cases classified as Castellvi's type II, disc herniation was found at the level above the LSTV in $83.4 \%$ of the cases, while herniated nucleus pulposus was observed in $75 \%$ (1). As far as the present case is concerned, type II was found in $86.3 \%$ of the studied patients with LSTV and low back pain in the study of Oyinloye et al. (14).

In addition, SSBO has been suspected as a predisposition factor for posterior disc herniation. The assumption is based on the hypothesis that the congenital defect could cause instability and lead to degenerative deformities (16). On the other hand, some studies support that the existence of SSBO is not responsible either for low back pain or disc herniation $(11,17)$. In an extended study among patients with low back pain, SSBO was detected in $8.5 \%$, although SSBO and LSTV were not associated with neurological findings (11). Preoperatively, SSBO may complicate the spinal anaesthesia during caudal epidural block, whereas ultrasonography and fluoroscopy may appear helpful (18). Moreover, the malformation may affect the surgical outcome during screw fixation, jeopardizing iatrogenic injury of the sacral nerves (19).

Correct segmentation and visualization of the spine is important before orthopaedic and neurosurgical procedures in the lumbar region. Radiographic evaluation may reveal the presence of SSBO preoperatively, while plain radiographs consist the optimum tool in order to discover the existence of LSTV and avoid intervening at the wrong level. Identification of the LSTV presence is possible on $30^{\circ}$ cranially angled anteroposterior radiograph of the lumbosacral region. An anteroposterior view that includes the thoracolumbar junction could be used in order to determine the vertebral level (20). In MRI imaging, several structures have been proposed to assist in vertebral segmentation. Paraspinal structures, such as the aortic bifurcation, inferior vena cava confluence, right renal artery, iliolumbar ligament, celiac and superior mesenteric artery origin have been proven helpful in determining the LSTV existence; in cases of L5 sacralization these structures are located more caudally, whereas in cases of S1 lumbarization the landmarks are positioned more cranially than expected (5).

\section{Conclusions}

As described, awareness of the variant morphology of the sacrum is essential for radiologists, anesthesiologists and spine surgeons. Congenital anomalies, such as the LSTV and the SSBO may cause confusion in the differential diagnosis or jeopardize the surgical outcome if not taken in mind. In the presented case, the two malformations co-existed in the same specimen, indicating the imperative need for precise knowledge of the potential anatomic variability of the region.

\section{References}

1. Castellvi AE, Goldstein LA, Chan DP. Lumbosacral transitional vertebrae and their relationship with lumbar extradural defects. Spine (Phila Pa 1976), 1984, 9(5): 493-5.

2. Eubanks JD, Cheruvu VK. Prevalence of sacral spina bifida occulta and its relationship to age, sex, race, and the sacral table angle: an anatomic, osteologic study of three thousand one hundred specimens, Spine (Phila Pa 1976), 2009, 34(15): $1539-43$.

3. Kamanli A, Genc H. Radiological abnormalities of the lumbosacral spine in young male individuals, J Back Musculoskelet Rehabil, 2002, 16(2): 91-4.

4. Otani K, Konno S, Kikuchi S. Lumbosacral transitional vertebrae and nerve-root symptoms, J Bone Joint Surg Br, 2001, 83(8): 1137-40

5. Lee CH, Park CM, Kim KA, et al. Identification and prediction of transitional vertebrae on imaging studies: anatomical significance of paraspinal structures, Clin Anat, 2007, 20(8): 905-14.

6. Apazidis A, Ricart PA, Diefenbach CM, Spivak JM. The prevalence of transitional vertebrae in the lumbar spine, Spine J, 2011, 11(9): 858-62.

7. Bertolotti M. Contributo alla conoscenza dei vizi differenzazione regionale del rachide con speciale riguardo all assimilazione sacrale della V. lombare, Radiol Med, 1917, 4: 113-4

8. Paraskevas G,Tzaveas A, Koutras G, Natsis K. Lumbosacral transitional vertebra causing Bertolotti's syndrome: a case report and review of the literature, Cases J, 2009, 2: 8320.

9. Santavirta S, Tallroth K, Ylinen P, Suoranta H. Surgical treatment of Bertolotti's syndrome. Follow-up of 16 patients, Arch Orthop Trauma Surg, 1993, 112(2): $82-7$

10. Ugokwe KT, Chen TL, Klineberg E, Steinmetz MP. Minimally invasive surgical treatment of Bertolotti's Syndrome: case report, Neurosurgery, 2008, 62(5 Suppl 2): ONSE454-5; discussion ONSE456.

11. Seçer M, Muradov JM, Dalgiç A. Evaluation of congenital lumbosacral malformations and neurological findings in patients with low back pain. Turk Neurosurg, 2009, 19(2): 145-8.

12. Taskaynatan MA, Izci Y, Ozgul A, Hazneci B, Dursun H, Kalyon TA. Clinical significance of congenital lumbosacral malformations in young male population with prolonged low back pain. Spine (Phila Pa 1976), 2005, 30(8): E210-3.

13. Elster AD. Bertolotti's syndrome revisited. Transitional vertebrae of the lumbar spine, Spine (Phila Pa 1976), 1989, 14(12): 1373-7.

14. Oyinloye OI, Abdulkadir AY, Babalola OM. Incidence and patterns of lumbosacral transitional vertebrae, in patients with low backpain in a Nigerian hospital. Nig Q J Hosp Med, 2009, 19(2): 95-9.

15. Milicić G, Krolo I, Anticević D, et al. Causal connection of non-specific low back pain and disc degeneration in children with transitional vertebra and/or Spina bifida occulta: role of magnetic resonance--prospective study, Coll Antropol, 2012, 36(2): 627-33.

16. Avrahami E, Frishman E, Fridman Z. Azor M. Spina bifida occulta of S1 is not an innocent finding, Spine (Phila Pa 1976), 1994, 19(1): 12-5. 
17. Magora A, Schwartz A. Relation between the low back pain syndrome and x-ray findings. 3. Spina bifida occulta, Scand J Rehabil Med, 2012, 12(1): 9-15.

18. Senoglu N, Senoglu M, Oksuz H. Landmarks of the sacral hiatus for caudal epidural block: an anatomical study, Br J Anaesth, 2005, 95(5): 692-5.
19. Srijit D, Shipra P. Spina bifida with higher position of sacral hiatus: a case report with clinical implications, Bratisl Lek Listy, 2007; 108(10-11): 467-9.

20. Hughes RJ, Saifuddin A. Imaging of lumbosacral transitional vertebrae, Clin Radiol, 2004, 59(11): 984-91.

Received: $22 / 02 / 2013$

Accepted in revised form: 25/09/2013

\section{Corresponding author:}

Dr. George Paraskevas, MD, PhD, Assistant Professor of Anatomy, Orthopedic Surgeon, Department of Anatomy, Medical School, Aristotle University of Thessaloniki, Post Box: 300, Post Code: 54124, Thessaloniki, Greece; e-mail: g_paraskevas (a)yahoo.gr 